\title{
Predictability Problems of Global Change as Seen through Natural Systems Complexity Description
}

\author{
1. General Statements \\ VLADIMIR V. KOZODEROV ${ }^{\mathrm{a}, *}$, VICTOR A. SADOVNICHII ${ }^{\mathrm{b}}$, SERGEY A. USHAKOV $^{\mathrm{b}}$ \\ and OLEG A. TIMOSHIN ${ }^{b}$ \\ ${ }^{a}$ Institute of Computational Mathematics Russian Academy of Sciences, Gubkine Street 8 , \\ 117333 Moscow, Russia; ${ }^{b}$ M.V. Lomonosov Moscow State University, 119899 Moscow, Vorobiovy Gory, MSU Russia
}

(Received in final form 2 June 1997)

\begin{abstract}
The overall problem of global change is considered as the mathematical discrete dynamics discipline that deals with the sets, measures and metrics (SMM) categories in information sub-spaces. The SMM conception enables to unify techniques of data interpretation and analysis and to explain how effectively the giant amounts of information from multispectral satellite radiometers and ground-based instruments are to be processed. It is shown that Prigogine's chaos/order theory and Kolmogorov's probability space are two milestones in understanding the predictability problems of global change. The essence of the problems is maintained to be in filtering out a "useful signal" that would spread from key regions of the globe as compared to their background. Global analysis, interpretation and modelling issues are outlined in the framework of incorrect mathematical problems and of the SMM categories, which contribute to solving the comparability problem for different sets of observations.
\end{abstract}

Keywords: Order and chaos, Imagery interpretation, Modelling

\section{INTRODUCTION}

Understanding of predictability problems of global change is considered by the world scientific community as having one of the highest priority in the context of international cooperation. Possible changes in climate, land use and composition of the atmosphere due to natural and anthropogenic influences are usually implied under the overall term of global change. Separate projects of the International Geosphere-Biosphere Programme/ IGBP (Global Change, 1992), World Climate Research Programme/WCRP (Morel, 1990), International Human Dimensions Programme on Global Environmental Change/IHDP (Moss, 1992) and other Programmes have been designed to solve the problems. The listed three Programmes (IGBP, WCRP, IHDP) are developed under the

*Corresponding author. 
International Council of Scientific Unions (ICSU), World Meteorological Organization (WMO) and International Council of Social Sciences (ICSS), respectively. Opportunities to study components of global change issues using satellite imagery, simulation techniques and data bases of specially arranged field campaigns have been realized within the Programmes. They reflect the-state-of-the-art in global change research.

Research objectives of the Programmes are to characterize and document variability and trends of the Earth's system through operational global observations, to understand the nature of key regulating parameters to identify the causal factors of observed variations and feedback processes, and to assess the predictable aspects of long-term variability and changes, including regional impacts, through the combined application of observations and models. The comprehensive research programmes that addresses these objectives should encompass the capability to measure and monitor key parameters, a strong field observation and data analysis stage to improve knowledge of key processes, a modelling activity to develop predictive capability for regional change, and an integrative analysis phase to evolve methods for impact assessments.

To achieve the ultimate goal of the Programmes, that is predictability of global change, is inconceivable without having in mind progress in mathematical disciplines of discrete dynamics for systems encountered in the natural and social sciences. But common sense and traditional principles are seen prevailing in the current projects realization. Mathematical aspects of information content assessments, discrete dynamics techniques and the evolutionary processes description are usually considered as something too abstract to be taken into account in the international projects. The conceptual endeavours, based on the mathematical aspects and their on-going improvements, seem to be not so attractive for researchers within the Programmes. The reasons of a certain kind of ignoring the mathematical frameworks to the detriment of common sense may be due to by the complication, connected with the general assertion of global change problems. Such conventional attitude to priorities in global change research gives way to allegedly more understandable results of simulation techniques. In fact, in the projects follow-up, the ultimate goal is somewhat forgotten, and numerical models (Global Circulation Model - GCM, SoilVegetation-Atmosphere Transfer - SVAT, Dynamic Global Vegetation Model - DGVM, etc.) are putting forward in the related studies (Asrar, 1989; Running et al., 1994). Satellite observations and remote sensing techniques are anticipated as serving mainly to run the models. These are models upon which main results of decision making are practically based, and it is clear that these models are necessary but not sufficient to solve problems of global change.

Meanwhile, temporal data sets of satellite observations would give an impetus for solving the predictability problems based on geoinformation models (Kozoderov, 1994), i.e. derived as a result of data sets processing using Geographical Information Systems (GIS), but not from taken in advance equations and numerical models constructed from these equations. This is a new class, called geoinformation models, that is wider in applications than pure simulation techniques. In climate research the predictability is usually associated with finding global responses of the Earth's climate system to anomalies of related processes in key regions of the World ocean and land surface. These anomalies, characterized by a certain kind of variability of thermodynamical parameters (atmospheric pressure, temperature, humidity) of the global system, are analyzed using temporal data sets of the Earth Radiation Budget (ERB) components from satellite observations (Marchuk et al., 1990).

The essence of the predictability problems is maintained in these studies to be in filtering out mathematically a "useful signal" that would spread from the key regions in the system as compared to their background. The concept of teleconnections, 
i.e. signal-to-noise ratios extraction from temporal data sets, is revealed in cross-correlations of the observed anomalies between the regions and other parts of the globe on the scales of the ERB interannual variability.

Another problem to be solved as a part of the overall predictability problem is in the necessity to realize that interpretation of remote sensing data using results of modelling of subsequent processes in the global system is not a mathematically correct procedure (Tikhonov and Arsenin, 1979) in the sense that even small disturbances of initial data could result in large changes in solutions of the inverse problem. A minimization procedure for a smoothing functional, that is equal to the sum of a stabilizing functional for the problem and a deviation measure between the operator of a hypothesized model and initial data of observations, is needed to make it possible to compare modelling and measurements data. This procedure is called as a regularization of approximate solutions finding for the corresponding functional equations, coupling modelling and measurement results (Tikhonov, 1982). Otherwise, the consistency of theoretical models application, while employing data of observations as their inputs, looks ambiguous without such procedures. This means in mathematical assertion of the comparability problem that data of modelling and observations could be in different sub-spaces, and taking into account stringent terms of sets, measures and metrics (SMM) (Kolmogorov and Fomin, 1976) with applied mathematical formalism may be the only way to understand to what extent sets of modelling and observation data are useful to solve the predictability problems.

In addition to the remarks concerning the conceptual SMM models application, one can say that besides a new sounding of such modelling techniques in data interpretation and analysis (Kozoderov, 1995), the SMM categories are meant to be a profitable tool in assessing state parameters of natural ecosystems using remotely sensed and ground-based data. On the eve of the Earth
Observing System (EOS) era (see Earth Observing System, 1993), including GCOS, GOOS and GTOS as the Global Climate, Ocean and Terrestrial Observing Systems, respectively, the scientific community mostly relies on traditional applications of the giant amounts of data to be acquired from the Systems. These applications are usually foreseen in diagnostic studies and numerical simulation techniques, which allegedly are to be fed by data of the observations.

In practice, tens of different instruments of the Systems (with various spectral and spatial resolutions) will give many Gigabytes of information, and no rigorous approach has confirmed that all these sets of data will be beneficial for employing in numerical models and other interpretations. In mathematical terminology this situation is understood that sets of observations and modelling are in non-overlapping sub-spaces, and additional steps are to be undertaken to give proofs concerning the effectiveness of the above Systems. Practical purposes of the Systems may look problematic unless the comparability problem of different data sets is elucidated by a proven scientific approach. Evolving the information and mathematical basis of the defined global change science, using the SMM categories in Kolmogorov's probability space (see part 2 of the publication), might be the only way to link different sets of measurements with their practical applications. This is an alternative to pure technological means in utilizing "the three-dimensional memory" while collecting and storing many Gigabytes of data within the EOS plans. The information content assessment and studies of redundance of sets of data will give a scientific tool for data processing, interpretation and analysis in the newly defined domain additionally to routine pattern recognition and scene analysis techniques.

Being adherent to Prigogine's ideas of dynamical systems description by equations of the evolutionary type (Prigogine and Stengers, 1986; 1994), we shall outline below major statements of the related theory to be known in global change, 
subject incentives of some IGBP projects to criticism because of their unreadiness to use the theoretical statements, and consider the natural targets complexity description problem as well as these targets variability and predictability of their alteration in the context of the global change science. In our attempts to give an explanation of the theoretical approach in terms of axiomatic prerequisites and rigorous mathematical definitions, we shall follow the proposed data sets concept in Kolmogorov's probability space along with the information measures and entropy metrics representation. These attempts are intended to link Prigogine's chaos/order theory and Kolmogorov's probability space as two milestones in understanding predictability problems of global change having in mind EOS data sets to be interpreted in terms of the above SMM categories.

Part 1 of the publication presents a general scope of mathematical aspects of the problems to be solved to understand what is the essence of the global change predictability: the specificity of remote sensing Earth sciences techniques to solve the inverse, mathematically incorrect, problems to unify data both of modelling and measurements; order and chaos as the main categories, the proposed conceptual models to be pertained to; global analysis, interpretation and modelling aspects of the overall predictability problems to use the mathematical procedures of multispectral satellite imagery processing as an example of new geoinformation models application.

Part 2 will embrace a description of our approach: Kolmogorov's probability space; properties of information measures that would enable to operate with information sub-spaces; imagery visualization procedures and the SMM categories; Markov's chains in the sub-spaces to find measures of any form of ordering on multispectral imagery; natural objects complexity and structure analysis techniques on sets of regular configurations of elements given by the information measures; the predictability problems in crosscorrelation techniques for anomalies of processes to be picked up by temporal sets of the imagery.

\section{BACKGROUND}

We have developed an information/mathematical approach that enables to solve the comparability problem of different sets of measurements relating to a particular natural object in terms of the SMMs (Sadovnichii, 1996). The approach is based on information measures and entropy metrics for any set of data using a mathematical expectance operator from specially interpreted probability distribution functions. The well-known Shannon's formula for the amount of information in any set of data gives a particular number to be calculated ("expected") as the sum, taken with the minus sign, of the products of a known probability distribution function from elements of the set and the logarithm (usually on base 2) of the function. The true sense of the information measure gets wider than simply the number calculated, if the formula is considered as a functional defined by the scalar product in the probability space that includes probability vectors, given by the distribution of the elements of the set, and other vectors, given by the non-linear logarithmic function from the distribution. The latter vectors can be called as the uncertainty vectors implying that no uncertainty exists in an event when the probability function is constant and equal to 1 (the certain event). Thus, additionally to the SMM categories we have to deal with representation of data in spaces, among which the distinction of Kolmogorov's space is not only in its containing sets of widely-used elementary events in mathematical sciences, but in using a specially defined algebra, i.e. this space gives "a rule of actions" with its elements along with its particular probability measures (Kolmogorov and Fomin, 1976). This space can be the $n$-measure Euclidean space for groups of numbers or elements, the linear normalized Hilbert's space with the scalar product on it, etc.

To outline scientifically the construction of the GCOS, GOOS and GTOS systems, our approach enables to merge the following interrelated blocks: Climate/Biosphere Models, Observation Systems, Geoinformation Systems and Predictability 
Problems. The opportunity to unify these blocks in the general context of global/regional change studies are determined by the proposed application of the information and thermodynamic properties of the entropy category. It reveals its dualism in the information content assessments for data of modelling/monitoring and in state parameters retrievals for natural targets using multispectral remote sensing images and ground measurements on test sites of the Earth.

The Climate/Biosphere block is designed to highlight how global and regional change problems are drawn up in terms of models, data and processes for natural media (the atmosphere, hydrosphere, land surface, biosphere). The $\mathrm{Ob}$ servation Systems block, linking modelling and monitoring quantities on various scales, serves to account for recommendations to optimization techniques based on certain criteria, value functions and information content metrics. The recommendations are due to the necessity to improve or make cost-effective the systems which are planned within the EOS and similar other programmes. The Geoinformation Systems block gives an explanation to what extent GIS-technologies, databases and information systems can be unified with general principles of data processing, related algorithms and procedures. The Predictability Problems block enables one to understand what can be determined from temporal data set analyses and interannual variability of multispectral satellite products and samples of ground measurements.

These our findings, which are designated to couple basic and applied research relating to discrete dynamics in nature and society, open up an opportunity to reduce uncertainties about current knowledge of natural processes by explaining differences between "pure random events" (chaotic distributions) and "an excessive ordering" to be characterized by uniform distributions (Prigogine and Stengers, 1986). As a result, tackling with the entropy functionals (conditional entropy, information divergence, mutual information, etc.), the effectiveness measures of global observing systems are derived in specific subject areas. Metrics is fitted in the relevant probability space to consolidate the unified description of data sets from different instruments. Besides that, we have shown that probabilistic grammatic rules and related computer languages on sets of regular configurations, extracted from sets of data using GIS, do contribute to a proper description of the complexity problem for structures of natural targets.

Thus, global change is a particular sphere of discrete dynamics application to study processes in nature and society using the proposed unification of scientific investigations in the SMM form for data analysis and interpretation in the related information sub-spaces. The conception of these sub-spaces as a composite part of widely used metrical spaces must become such a universal means to assess the state of the objects under study while processing data sets that would carry information about objects using computer work stations. Though not terminate in applications, the terminology of the functional analysis discipline (for example, linear shells or compactness of sets as well as metrical spaces or their completeness) are routinely coming into software of existing work stations for data sets processing. A pair of quantities is understood as usual under the metrical space, that consists in a set of elements (pixels for imagery) of a particular structure and in a distance, i.e. a single, non-negative and real function to be defined for two optional elements of the given set and satisfied the known axioms of "the zero element existence, symmetry and triangle" (Kolmogorov and Fomin, 1976).

The idea to employ the probability space representations is vital for us to help unify processing of different data sets: of measurements in remote sensing Earth sciences, of questionnaires concerning regional data on the health care and infrastructure, of indicators of possible change using ground observations, etc. In any case, understanding of the relevant probability distributions is needed in the form of order and chaos measures to be extracted from the specific sets of data. In this respect we can say about applications 
of our approach as a new step in global change science operating with discrete sets of data in information sub-spaces.

\section{ORDER AND CHAOS CATEGORIES}

Irreversibility of processes in the natural world is one of its fundamental properties. The important attainment of Prigogine's theory (see Prigogine and Stengers, 1986; 1994) is that the theory has demonstrated by non-contradictory evidence the universal entropy increase for spontaneous natural processes as a reflection of objective properties of instability in dynamic systems (the upper limit of which are chaotic systems). We do not dwell here on thermodynamic and information properties of the entropy (Kozoderov, 1995), which are known to be formally equivalent (Volkenstein, 1986). Our main interest is concerned the information entropy and the consequent measures of uncertainty described by it.

Classical thermodynamics is unable to explain processes of energy and mass exchange in open biological and other systems. The rate of the entropy production in stationary positions to be reached as a result of internal irreversible processes in such systems is equal to its minimal positive meaning. So, the entropy is not only unlimited "slipping down" to the stationary position without constructing any organized structure, but it serves as a source of a certain measure of order emerging in the systems. The classical description of the systems behaviour in terms of trajectories in the phase space, containing spatial coordinates and time, turns out to be not sufficient for chaotic systems, for which behaviour cannot be predicted similarly to the classical case. The constructive role of chaos, that leads to the rise of local ordering and to decrease of natural systems entropy whilst its overall tendency is to be increased, is among basic principles in Prigogine's theory.

Overall findings of the theory are in gaining proofs that producing the chaos is an objective property of dynamic systems. Such systems would characterize the evolution of nature and society. The properties of the systems can be analyzed by an irreducible probabilistic description of these systems in generalized spaces, where complex eigen values of mathematical operators in the equations of the evolutionary type give information about processes of decay, damping and irreversibility in addition to classical descriptions of the dynamic processes. These findings of Prigogine's theory would entail a necessity of utilizing mathematical axiomatics of probability theory, information content assessments and entropy characterization to employ the basic ideas of dynamical systems behaviour for representing sets of remote sensing data as units of the relevant metrical spaces. These treatments, in turn, would unify the process of the natural objects complexity description, comparability of their images and finding their variability using temporal data sets of their sub-sequent imagery.

Saying about chaotic systems as having the upper level of instability, when a phase trajectory loses its sense through a characteristic interval called "Ljapounov's time", we are able to observe changes on multispectral imagery with time by representing sets of data in metrical spaces. This our opportunity to visualize structures of natural objects on their imagery would enable to interpret the emergence "of particular points" of possible bifurcation as they belong to "rules of their systematization" using special computer languages while processing sets of the imagery. Chaotic systems are known to "forget" their initial conditions in their evolution after the period of Ljapounov's time. This definition has allowed scientists to solve many problems of natural development and evolution while satisfying the following main requirements of chaos origin:

- the existence of irreversible processes that is expressed in the symmetry violation between the past and future events;

- the necessity to introduce "the event" category as permitting descriptions only in terms of probabilities; this category cannot be derived 
from any deterministic law, reversible in time or not;

- some events must have the ability to change the course of evolution, i.e. the evolution is to be definitely "unstable" and characterized by mechanisms that make some events initial points of new developments and a new global order.

Contrary to closed or isolated (without interaction with their environment) deterministic systems, characterized by classes of stable solutions in the relevant differential equations, new possibilities enter into consideration in "the open world" dealing with the irreducible probabilistic laws. The irreducibility is the direct consequence of the instability of the chaotic systems. There is a threshold for open systems in hydrodynamics, chemical kinetics and other sciences, from which the systems behaviour gets radically changed. The result is in emerging "dissipative structures" (Kurdyumov, 1982). These are understood as having "an organized behaviour" resulting from interactions between two opposite processes in the non-equilibrium thermodynamics: dissipation and order. Even no new ordering force is induced into the system, far from the equilibrium position a spontaneous violation of the spatial symmetry can emerge in the system under coherent collective motions because of non-linear properties of the strongly non-equilibrial situations. These are situations that result in such effects as a sensitivity of the solutions to initial conditions, instability and bifurcations, i.e. giving rise to many ways of a particular system developing near these points of its phase trajectories.

The thermodynamic equilibrium state is called "an attractor", the isolated system is directed to, that consists of many billions of particles, but might be described by only a small number of parameters such as temperature and pressure. The attractors are synonymous with the stability and reproducibility categories. However, attractors with a fractional dimension ("strange attractors") cannot be described in terms of simple geometrical objects and thus require that the cause and consequence categories, known in deteministic relations, be replaced by their new properties.

The dissipative chaos is a phenomenon that arises in unstable dissipative structures for greatly non-equilibrial processes when the regime of the strange attractor gets chaotically unstable, i.e. when the distance between two points of the system, initially very small, is exponentially enhanced with time. The possibility of predicting the behaviour of such systems is vague, differing from small fluctuations in the case of "the deterministic chaos," which can be described in terms of expected correlations in temporal data sets, contrary to "a completely random process." The latter is characterized by infinitely large dimensions, and its temporal evolution is governed by internal random phenomena, but not global dynamics or an attractor.

These major statements of Prigogine's theory enable us to formulate the predictability problems of global change in a linear cross-correlation approach when the possibility of predicting the dynamic behaviour of the Earth's geophysical/ biogeochemical/climate system remains real. We originate our research from the fact that the ultimate goal of understanding the predictability problems in the system can be achieved by multidisciplinary studies using global monitoring systems, regional networks and models merging scientific and technological issues. Therefore, the predictability theory is outlined in part 2 of this publication based on temporal data sets analysis for remote sensing imagery of various spatial resolutions including global and regional scales. In fact, these goals can be reached provided that rules of information measures application are determined to represent sets of data in metrical sub-spaces. Only for the sub-spaces, mathematically formalized by definitions of sets and a distance between two points in them, it is possible to use common measures of comparability, ordering and calculations.

Some extractions from the information theory in part 2 are assumed to use the particular form of the mathematical expectance operator for matching 
sets of data in information sub-spaces instead of Hamilton's operator used in Prigogine's publications for the classical approach. Our conviction to yield a new quality in understanding of predictability problems of global change using the information amount assessments is strengthened by the fact that evolving imagery visualization procedures in terms of the entropy measures, we can motivate what is the "most interesting" in natural observable structures being exposed to natural and anthropogenic changes. Analyzing the commensurate "contextually coherent structures" of natural targets, we are able to find what is essential in a particular event, given by a sequence of symbols on multispectral imagery. This is a possible way to remove the major vulnerability of the algorithmic theory of information, which is due to by the supposition that the structure under consideration is not infinite. Thus, an opportunity is opened up to elucidate relations between the initial sense of the structure formation and means to describe its variability. Our approach enables to make the complexity description of natural targets behaviour using contextual computer languages.

Before going to information measures consideration, let us shortly outline main tendencies in data analysis, interpretation and modelling. The proposed conceptual framework of global change has turned out not yet to be of particular importance in global change science due to lack of its proper mathematical influence on existing studies.

\section{GLOBAL ANALYSIS, INTERPRETATION AND MODELLING IN INTERNATIONAL PROGRAMMES}

Possibilities to solve global change problems are to great extent associated with global system modelling, parameterizations of natural processes in the relevant numerical models and integration of consequent sub-system models. In particular, the IGBP Task Force for Global Analysis, Interpretation and Modelling (GAIM) is conceived by its initiators as directed "toward establishing the research capabilities necessary for developing the models and understanding needed for construction of integrated biogeochemical Earth system models" (Research GAIM, 1997). This is only one part of the multidisciplinary problem to understand the predictive capabilities of global change. Its second part is concerned the role of regular observations and current measurements in the studies. How can these parts be coupled? This question is behind the GAIM and similar other projects consideration, though in light of the EOS era the second part seems to be more actual to date than the first one.

An impression can be gathered that the GAIM and other IGBP projects (for instance, International Global Atmospheric Chemistry - IGAC, Global Change and Terrestrial Ecosystems - GCTE, Biospheric Aspects of the Hydrological Cycle-BAHC, Land Use/Cover Change-LUCC, etc.) participants come consciously away from vital problems concentrating mostly their attention on modelling and partly on analysis problems, but not on that of data interpretation (see, for example, Natural Disturbances ..., 1996). Not denying attempts within the GAIM to create sub-system models of the global biogeochemical system, we are saying here about conceptual models to describe complex non-linear phenomena within the global system using mathematical formalism of probability spaces, sets of data, information measures, entropy metrics, etc.

We see our purpose to fill in the gap between data of modelling and monitoring having in mind the challenge in gaining the ultimate goal - to understand the predictability problems of global/ regional change. Giving tools to different data sets comparability, the conceptual models would enable to solve the problems. Data interpretation in the GAIM Task Force should include mathematically stable retrieving of state parameters for natural targets within a concurrent class of models using particular solutions of incorrect problems. These are other procedures of remote sensing data processing than are envisioned by the GAIM.

The general direction in the GAIM type projects implementation is in solving direct problems, i.e. 
in obtaining results of related hypothesized theoretical models. Practical criteria of making conclusions, concerning global/regional change, are determined by solutions of inverse problems that are designed to account for the quality of one or another model from sets of the hypothesized classes of comparable models. The questions are put while solving the inverse problems: (i) whether an individual model in an accepted class exists or not, theoretical consequences and particular observations for this models are within the accuracy of the observations (the comparability problem is set up for the class of the models used and the observations for a particular natural target); (ii) if the class of the models is comparable with observations, then to realize an interpretation of the particular set of observations, i.e. to explain the value of the individual theoretical model among the accepted class of models under comparison.

\section{CORRECT AND INCORRECT PROBLEMS}

The final purpose of the global Earth system models construction will look vague without the above basic statements of simulation techniques. If we denote by $z$ an approximate model of a natural object, then it is usually represented by a set of numerical parameters, functions, etc. so that all other models of the same type from the class $Z=\{z\}$ of the comparable models are considered identical, provided all their characteristics for the models are completely coincident. Hence, a deviation measure between two models $z_{1}$ and $z_{2}$, denoted as $\rho_{Z}\left(z_{1}, z_{2}\right)$, can be defined (Tikhonov, 1982) being considered as elements of the given metrical space (Kolmogorov and Fomin, 1976). In practice, it means that we are eligible to separate correct and incorrect problems, the first are to satisfy the requirements of their solutions existence and uniqueness as well as stability of the solution relative to disturbances of initial data, the second are called when at least one from these three conditions are not satisfied.
If $u$ is a theoretical value of observational inputs to be found in accordance with the idealized model $z$ and $A$ is the mathematical operator that determines a relationship between characteristics of the $z$ model and input data $u$ so that $u=A z$, then the similar deviation measure $\rho_{U}\left(u_{1}, u_{2}\right)$ can also be defined in the class of possible input curves of observations $U=\{u\}$. The form of the $A$ operator is usually established owing to the theory that serves as a basis for interpreting data of observations. In specific applications while interpreting remote sensing data, the following integral Fredholm equation of the first kind, well-known in mathematical analysis, has to be solved:

$$
A z=\int_{a}^{b} K(x, s) z(s) \mathrm{d} s=u(x), \quad c \leq x \leq d,
$$

where the equation kernel $K(x, s)$ has to be a continuous function $c \leq x \leq d, a \leq s \leq b ; z(s)$ is the function to be found for the object under study; $u(x)$ is a known function given by the particular experiment.

An explicit form of the $A$ operator while processing remote sensing data is usually associated with the radiation transfer theory (Kondratyev et al., 1992). But the problem of the related observations interpretation is set up as that of finding solutions of the following equation:

$$
A z=u^{*}
$$

that serves for a pattern recognition of the object under study using approximate input data of particular observations $u^{*}$. The assertion of the problem is mathematically inconsistent since $u^{*}$ is a product of a particular measurement for an object, with inevitable complications due to the measurement inaccuracy. The latter could result in possible absence of any solution that would satisfy the equation in the $Z$ class of all permissible solutions.

If the $A$ operator is explicitly known and $\delta$ is the inaccuracy amplitude for the $u^{*}$, so that for the $u^{-}$ "precise" measurements the deviation measure can 
be written

$$
\rho_{U}\left(u^{*}, u^{-}\right) \leq \delta
$$

then the inverse problem is set up as that of the following inequality solution finding:

$$
\rho_{U}\left(A z, u^{*}\right) \leq \delta \quad(z \in Z)
$$

Elements $z^{\delta}$, satisfying this inequality, can be nominated as formally comparable with the $u^{*}$ values. Denoting via $Z^{\delta}$ the set of all $z^{\delta}$, comparable with these values

$$
Z^{\delta}=\left\{z^{\delta} \in Z: \rho_{U}\left(A z^{\delta}, u^{*}\right) \leq \delta\right\}
$$

one can obtain classical solutions of the inverse problem.

If the set of $Z^{\delta}$ is empty, i.e. models comparable with a set of observations in the $Z$ class are absent, this means that these models from $Z$ are too rough and cannot describe the object under study with the given accuracy. Thus, it is necessary to widen the $Z$ class of models taking an entire set of extending classes of the hypothesized models $Z_{1}, Z_{2}, \ldots, Z_{n}$ until the following inequality (that would characterize the infimum of the deviations between the theoretical values of $A z$ and data of measurement $u^{*}$ known approximately) is satisfied:

$$
\inf _{z \in Z_{n}} \rho_{U}\left(A z, u^{*}\right) \leq \delta
$$

i.e. until a comparable (consistent) model of the object is found.

In the case, when $Z^{\delta}$ is not empty and contains different models, the formal comparability is not sufficient to identify the related model of the object using only observations, given with their accuracy, without additional information. It means that even the above equation $A z=u^{*}$ does have a solution, the solution of the stated inverse problem can be unstable in the sense that even small variations in data of observations will result in large variations in the solutions of the whole problem. This equation may not have any solution in its classical sense, and in this case Mean Square Techniques (MST) are usually implied to find a generalized solution, which is understood as such a model realization $z_{0} \in Z$ that

$$
\rho_{U}\left(A z_{0}, u^{*}\right)=\inf _{z \in Z_{n}} \rho_{U}\left(A z, u^{*}\right)
$$

The MST improvements are known to solve the problem concerning the existence of a formally comparable model, provided the model $z_{0 \eta}$ can be found that would "almost completely minimize" the deviation with the accuracy $\eta$, such as $\delta_{0}+\eta<\delta$, where

$$
\delta_{0}=\inf _{z \in Z} \rho_{U}\left(A z, u^{*}\right)<\delta
$$

The MST solutions are often used in remote sensing data processing, but this is an example of unstable solutions in the sense defined above. Stable algorithms of observational data interpretation are given by regularization techniques (Tikhonov and Arsenin, 1979).

If the given $Z^{\delta}$ set of the formally comparable solutions is too wide, then it is necessary to establish a selection principle how to choose a most valuable model $\bar{z} \in Z^{\delta}$ from the point of view of its applicability for interpretation purposes. The choises are given by the regularization techniques. The principle of "minimal complexity" of a model, being selected from sets of similar models to be comparable in accordance with data observation accuracies, is often laid down into the selection process. The complexity functionals $W[z]$ should be non-negative, continuous and satisfying to the condition that the set $Z_{c}=\{z \in Z: W[z] \leq c\}$ is compact in the $Z$ space. The compactness implies that from any sequence of objects in the metrical space a new sub-sequence can be selected that should converge to an element in $Z$. As a result, a normal solution of the problem is given by such an element $\bar{z}^{\delta} \in Z^{\delta}$, which would minimize the complexity functional

$$
W\left[\bar{z}^{\delta}\right] \leq W[z] \quad \text { for all } z \in Z^{\delta}
$$


i.e. $\bar{z}^{\delta}$ is the least complex model from the class of all models comparable with observations according to the accuracy category.

There are theorems of uniqueness of the $\bar{z}^{\delta}$ determination under sufficiently general properties of the $W[z]$ functional, of stability of the $\bar{z}^{\delta}$ model selection process relative to small perturbations of the $\bar{u}^{\delta}$ observations as well as of convergence of the normal solution to exact when the observation accuracy increase is not restricted (Tikhonov and Arsenin, 1979). Thus, $\bar{z}^{\delta}$ is a stable regularized solution of the equation $A z=u^{* \delta}$.

There are several techniques of the optimal functional $W[z]$ selection. This example of global analysis, interpretation and modelling shows that the problems to be put in the GAIM are more complicated than considered by the Task Force. Creating global sub-system models looks like typical exercises in convenient simulations without studying the complete set of the EOS and other systems data interpretation for the global purposes. Part 2 of the publication will be devoted to information and complexity measures, their description and use to understand the predictability problems of global change.

\section{References}

Asrar, G. (Ed.) (1989). Theory and Application of Optical Remote Sensing. John Wiley \& Sons, New York, USA, p. 890.

Earth Observing System (EOS): Reference Handbook (1993). NASA, Washington D.C., USA, p. 145.

Global Change: Reducing Uncertainties. The International Geosphere-Biosphere Programme: A Study of Global Change (1992). International Council of Scientific Unions, Stokholm, Sweden, p. 42.

Kolmogorov, A.N. and Fomin, S.V. (1976). Elements of Theory of Functions and the Functional Analysis. Nauka Publ., Moscow, p. 544.
Kondratyev, K.Ya., Kozoderov, V.V. and Smokty, O.I. (1992). Remote Sensing of the Earth from Space: Atmospheric Correction. Springer-Verlag, Heidelberg, p. 478.

Kozoderov, V.V. (1994). Generalized assertion of problems of state parameter assessments for natural targets using optical remote sensing from space. Earth Observations and Remote Sensing 6, 51-67.

Kozoderov, V.V. (1995). A scientific approach to employ monitoring and modelling techniques for Global Change and Terrestrial Ecosystems and other related projects. J. Biogeography 22, 927-933.

Kurdyumov, S.P. (1982). Eigen functions of the non-linear media combustion and constructive laws of finding an organization in the media. In: Problems of Mathematical Physics and Computational Mathematics. Nauka Publ., Moscow, pp. 217-243.

Marchuk, G.I., Kondratyev, K.Ya. and Kozoderov, V.V. (1990). Radiation Budget of the Earth: Key Aspects. Nauka Publ., Moscow, p. 224.

Morel, P. Climate Change: A Scientific Review Presented by the World Climate Research Programme (1990). World Meteorological Organization - International Council of Scientific Unions, Geneva, Schwitzerland, p. 36.

Moss, R.H. (1992). Human dimensions of Global Environmental Change Programme $(H D P)$. IGBP - Global Change, Report No. 22, Stokholm, Sweden, p. 114.

Natural Disturbances and Human Land Use in Dynamic Global Vegetation Models (1996). IGBP - Global Change, Report No. 38, Stokholm, Sweden, p. 49.

Prigogine, I. and Stengers, I. (1986). Order out of Chaos. Bantam, New York, p. 432.

Prigogine, I. and Stengers, I. (1994). Time, Chaos, Quantum. Progress Publ., Moscow, p. 266.

Research GAIM (1997). The Newsletter of the Global Analysis, Interpretation, and Modelling Task Force. IGBP-GAIM, No. 1, Stokholm, Sweden, p. 20.

Running, S.W., Loveland, T.R. and Pierce, L.L. (1994). A vegetation classification logic based on remote sensing for use in global biogeochemical models. Ambio 23, 77-81.

Sadovnichii, V.A. (Ed.) (1996). Remote Sensing Earth Sciences: Information and Mathematical Principles. Authors: Kozoderov, V.V., Kosolapov, V.S., Timoshin, O.A. et al., Moscow State University Publ., Moscow, p. 572.

Tikhonov, A.N. (1982). Mathematical physics and data processing of observations. In: Problems of Mathematical Physics and Computational Mathematics. Nauka Publ., Moscow, pp. 292-301.

Tikhonov, A.N. and Arsenin, V.Ya. (1979). Methods of Incorrect Problems Solution. Nauka Publ., Moscow, p. 285.

Volkenstein, M.V. (1986). Entropy and Information. Nauka Publ., Moscow, p. 192. 


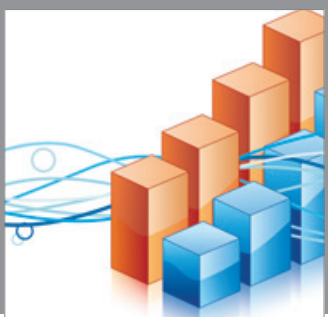

Advances in

Operations Research

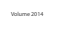

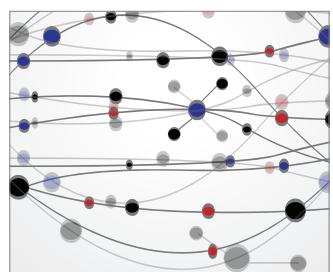

\section{The Scientific} World Journal
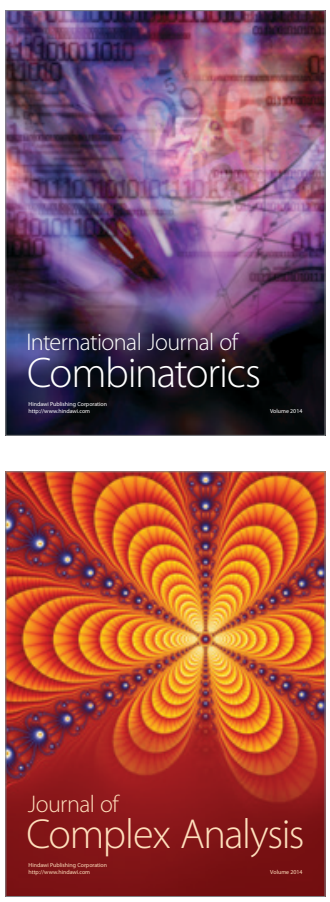

International Journal of

Mathematics and

Mathematical

Sciences
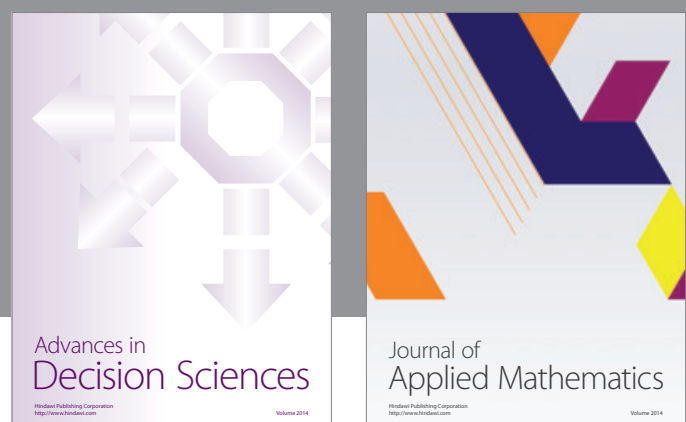

Journal of

Applied Mathematics
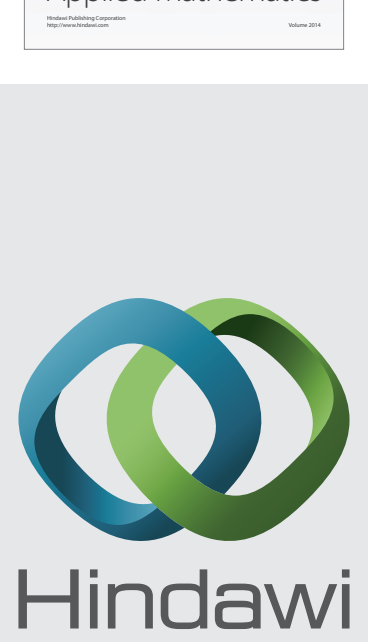

Submit your manuscripts at http://www.hindawi.com
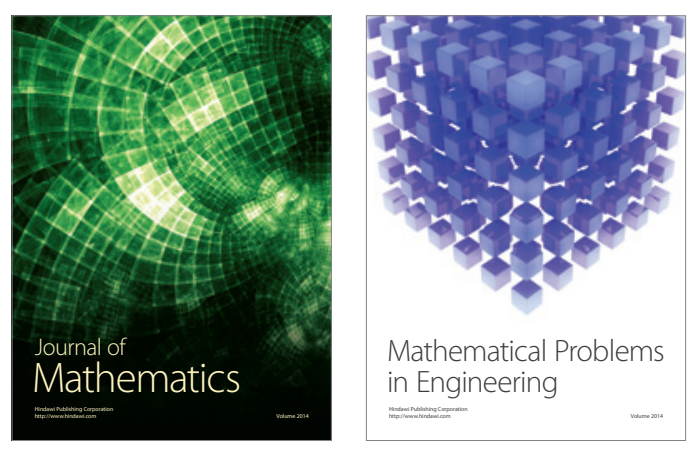

Mathematical Problems in Engineering
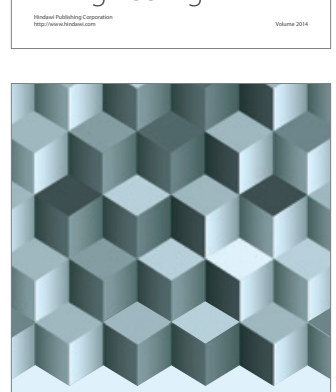

Journal of

Function Spaces
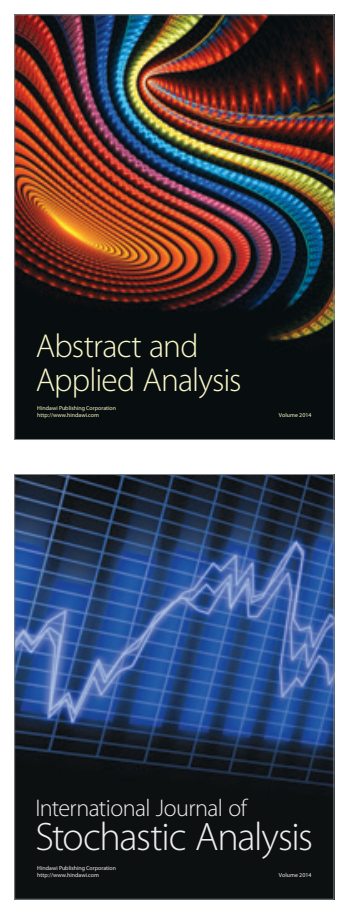

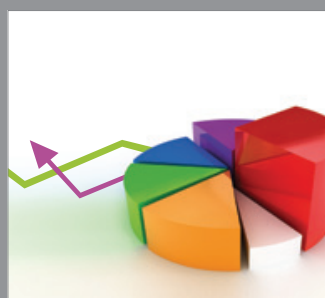

ournal of

Probability and Statistics

Promensencen
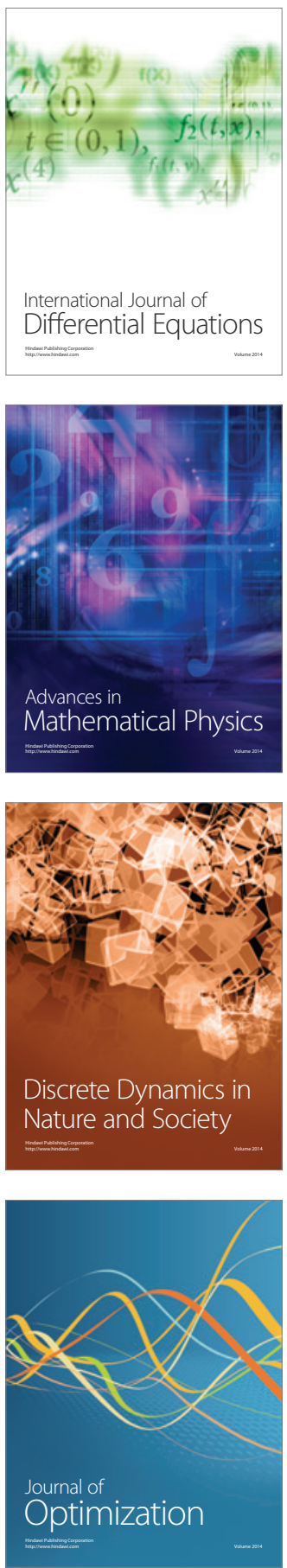